\title{
Attenuation of liver fibrosis by herbal compound 861 via upregulation of BMP-7/Smad signaling in the bile duct ligation model rat
}

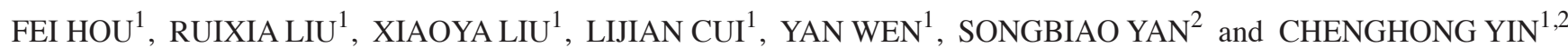 \\ ${ }^{1}$ Department of Infection, Beijing Friendship Hospital, Capital Medical University, Beijing 100050; \\ ${ }^{2}$ Department of Internal Medicine, Beijing Obstetrics and Gynecology Hospital, \\ Capital Medical University, Beijing 100026, P.R. China
}

Received April 29, 2015; Accepted March 8, 2016

DOI: $10.3892 / \mathrm{mmr} .2016 .5071$

\begin{abstract}
Herbal compound 861 (Cpd 861) exerts an anti-fibrotic effect in patients with hepatic fibrosis; however, the anti-fibrotic mechanism has yet to be fully elucidated. The present study aimed to explore the mechanistic basis for the anti-fibrotic effect, with a focus on bone morphogenetic protein 7 (BMP-7)/Smad signaling in a bile duct ligation (BDL)-induced liver fibrosis rat model. Following the induction of hepatic fibrosis, rats induced by BDL were treated with $9 \mathrm{~g} / \mathrm{kg}$ Cpd 861 daily or an equal volume of saline for 28 days. Serum samples were prepared for monitoring the levels of alanine transaminase, aspartate transaminase and total bilirubin, and direct bilirubin analyses and liver samples were used to investigate gene expression, protein localization and protein expression analysis using real-time quantitative polymerase chain reaction, immunohistochemistry and western blotting. The results revealed the attenuation of liver fibrosis by Cpd 861 in the histological and biochemical experiments. BMP-7 and phospho (p)-Smad1/5/8 were localized predominantly in the cytoplasm of hepatocytes. In comparison with the saline-treated BDL rats, Cpd 861 markedly upregulated the gene expression of BMP-7 and Smad5, as well as the protein expression of BMP-7 and Smad1/5. In addition, p-Smad1/5/8 protein expression was markedly increased by $\mathrm{Cpd} 861$ in the BDL model. These results indicated that Cpd 861 alleviates hepatic fibrosis possibly via the upregulation and activation of BMP-7/Smad signaling in hepatic fibrotic rats.
\end{abstract}

Correspondence to: Professor Chenghong Yin, Department of Infection, Beijing Friendship Hospital, Capital Medical University, 95 Yong'an Road, Beijing 100050, P.R. China

E-mail: modscn@126.com

Professor Songbiao Yan, Department of Internal Medicine, Beijing Obstetrics and Gynecology Hospital, Capital Medical University, 251 Yaojiayuan Road, Chaoyang, Beijing 100026, P.R. China E-mail: sbybjfcyy@126.com

Key words: Cpd 861, liver fibrosis, BMP/Smad signaling

\section{Introduction}

Hepatic fibrosis represents a wound-healing response to liver injury, resulting from a number of chronic liver diseases, including viral and autoimmune hepatitis, biliary obstruction and alcohol consumption. Globally, hepatitis B, hepatitis C and alcohol consumption have been the leading causes of hepatic fibrosis, although recently, the increasing prevalence of obesity and metabolic syndrome has further increased the incidence of liver fibrosis (1). At the end-stage of chronic liver diseases, hepatic fibrosis may result in cirrhosis, portal hypertension, liver failure and hepatocellular carcinoma (2). Chronic liver diseases and cirrhosis are important causes of morbidity and mortality worldwide (1).

The occurrence of hepatic fibrosis is a dynamic process, which is intimately associated with the abnormal structure and dysfunctions of the liver, involving cytokines and such cells as hepatocytes, hepatic stellate cells (HSCs), Kupffer cells and hepatic sinusoidal endothelial cells. Progression to hepatic fibrosis is characterized by an excessive deposition of extracellular matrix (ECM), including such components as collagens, glycoproteins, mucopolysaccharides and proteoglycans (3). The ECM is predominantly generated by HSCs, whose activation and proliferation fulfill a major role in the development of hepatic fibrosis. In normal liver, HSCs exhibit a star-like morphology, are located in the space of Disse surrounding the sinusoids as pericytes, and store vitamin-A-associated lipid droplets. Upon activation, HSCs undergo a phenotypic transformation into myfibroblast-like cells, as demonstrated by the expression of $\alpha$-smooth muscle actin ( $\alpha$-SMA) and the dysregulation of collagens, matrix metalloproteinases (MMPs) and tissue inhibitor of metalloproteinase 1 (TIMP1), processes which are largely attributed to the increased deposition of ECM. Among the members of the collagen family, collagens I and III are predominantly associated with fibrogenesis, and increases in their expression levels occur concomitantly with the progression of liver fibrosis.

An emerging body of evidence has indicated that increasing levels of transforming growth factor (TGF)- $\beta 1$, a major profibrogenic cytokine, induce intracellular signaling events, resulting in excess matrix protein deposition and an 
inhibition of the synthesis of ECM-degradation enzymes (4-6). Bone morphogenetic proteins (BMPs) are members of the TGF- $\beta$ family (7), which exert their biological functions via interaction with types I and II serine/threonine kinase receptors. Signals from the types I and II receptors are subsequently transmitted to the downstream substrates, including Smads $(8,9)$. Once BMP ligands are stimulated, R-Smads are phosphorylated and form a complex with Co-Smads (Smad4), which are subsequently translocated into the nucleus to trigger the expression of target genes (9).

However, BMP-7 possesses anti-inflammatory and anti-fibrotic properties in the fibrotic model (10). BMP-7 has been demonstrated to inhibit TGF- $\beta 1 /$ Smad signaling $(11,12)$. BMP-7 was revealed to enhance the phosphorylation of Smad1/5/8 [phospho (p)-Smad1/5/8], resulting in the suppression of the profibrogenic effect of TGF- $\beta 1$ (13). Therefore, BMP-7/Smad signaling has attracted special attention for its anti-profibrogenic effects via the negative regulation of TGF- $\beta 1 /$ Smad signaling.

Currently, multiple treatment strategies aimed at alleviating the inflammatory necrosis of hepatocytes and ameliorating the severity of hepatic fibrosis have been employed, including the inhibition of inflammation, targeting the host immune response, HSC activation and ECM synthesis. Herbal compound 861 (Cpd 861) is an extract of several mixed Chinese herbs, including red sage (Salvia miltiorrhiza), Astragalus membranaceus, Radix Bupleuri, Spatholobus suberectus, Szechwan Lovage Rhizome, nut grass (Cyperus rotundus) and red paeonia. Clinical trials have revealed that $\mathrm{Cpd} 861$ is able to markedly ameliorate clinical symptoms in patents with hepatic fibrosis, and experimental observations demonstrated that the formula reverses the progression of liver fibrosis in rat models (14). Further studies have lent support to the hypothesis that Cpd 861 inhibits the expression of TGF- $\beta 1$, the proliferation and activation of HSCs and the progression of hepatic fibrosis $(15,16)$. However, the anti-fibrotic molecular mechanism which underpins the action of Cpd 861 has yet to be fully elucidated. The present study aimed to further determine whether Cpd 861 attenuates hepatic fibrosis via the upregulation of BMP-7/Smad signaling.

\section{Materials and methods}

Animal grouping and treatment. Animal care and experimental procedures were approved by the Ethics Committee of Beijing Friendship Hospital, Capital Medical University (Beijing, China). A total of 18 male SPF Wistar rats, weighing 180-220 g, were obtained from the Laboratory Animal Center of Chinese Academy of Medical Sciences (Beijing, China). All animals were maintained under pathogen-free conditions and allowed unlimited access to food and water. Following acclimation, the rats were randomly assigned to three groups containing six rats each: The sham-operation group, the bile duct ligation (BDL) model group and the Cpd 861-treated group. Bile duct ligation (BDL) and sham operation were performed as described previously $(17,18)$ under general anesthesia induced by intraperitoneal injection of sodium pentobarbital $(40 \mathrm{mg} / \mathrm{kg}$; Sigma-Aldrich, St. Louis, MO, USA). Following midline laparotomy, the common bile duct was identified, twice ligated with 6.0 silk sutures (19) and sectioned between the ligatures. The abdomen was subsequently closed, and rats were allowed to recover under a heating lamp. The animals in the Cpd 861-treated group were intragastrically administered $9 \mathrm{~g} / \mathrm{kg}$ Cpd 861 (Beijing Tcmages Pharmaceutical Co., Ltd., Beijing, China) daily for 28 days, and the remaining two groups were intragastrically administered with an equal volume of physiological saline. Following the treatment, animals were sacrificed. Following anesthesia by intraperitoneal injection of sodium pentobarbital $(40 \mathrm{mg} / \mathrm{kg})$, midline laparotomy was performed and the right lobe of the liver was identified and collected from the animals. Prior to sacrification of the rats, blood samples were collected from the heart.

Histological examination. Liver tissues were fixed in $4 \%$ paraformaldehyde, paraffin-embedded, and sectioned at $4 \mu \mathrm{m}$. Histological changes were assessed using hematoxylin-eosin (H\&E) and Masson staining (Bestbio Co., Shanghai, China). Images from the different groups were captured using a microscope (Leica DM6000B; Leica Microsystems GmbH, Wetzlar, Germany).

Detection of serum biochemical indicators. Serum was isolated by centrifugation at $1,811 \mathrm{x}$ g for $5 \mathrm{~min}$, and subsequently used for alanine transaminase (ALT), aspartate transaminase (AST), total bilirubin (TBIL) and direct bilirubin (DBIL) analyses using an automatic biochemistry analyzer (Abbott Laboratories, North Chicago, IL, USA), according to the manufacturer's protocol.

Reverse transcription-quantitative polymerase chain reaction $(R T-q P C R)$. Total RNA was isolated from the preserved liver tissues using TRIzol ${ }^{\circledR}$ reagent (Invitrogen; Thermo Fisher Scientific, Waltham, MA, USA), according to the manufacturer's protocol. RNase-free deoxynuclease (Promega Corp., Madison, WI, USA) was used to eliminate any contaminating genomic DNA. The first cDNA strand was synthesized by reverse transcription reaction in a $20 \mu \mathrm{l}$ sample containing $4 \mu \mathrm{lgCl}_{2}$, $3 \mu \mathrm{l}$ 10X RT buffer, $2 \mu \mathrm{l}$ deoxynucleotides mixture, $0.5 \mu \mathrm{l}$ recombinant ribonuclease inhibitor, $0.6 \mu 1$ avian myeloblastosis virus reverse transcriptase, $1 \mu$ l Oligo dT-Adaptor primer and $5 \mu \mathrm{g}$ RNA sample (A3500; Promega Corp.). The RT reaction product was subsequently used for the amplification of cDNA using the SYBR ${ }^{\circledR}$ Mastermix reaction system (Thermo Fisher Scientific) comprising, in a total sample of $10 \mu \mathrm{l}, 1 \mu \mathrm{l}$ upstream primers, $1 \mu \mathrm{l}$ downstream primers, $1 \mu \mathrm{l}$ cDNA and $7 \mu \mathrm{l}$ diethylpyrocarbonate-treated water. The primer sequences are listed in Table I, and the housekeeper gene, glyceraldehyde-3-phosphate dehydrogenase (GAPDH), was used as an internal control.

The PCR protocol was as follows: The mixture was first denatured at $95^{\circ} \mathrm{C}$ for $10 \mathrm{~min}$, followed by 40 cycles at $95^{\circ} \mathrm{C}$ for $15 \mathrm{sec}$ and $64^{\circ} \mathrm{C}$ for $30 \mathrm{sec}$, and subsequently followed by $95^{\circ} \mathrm{C}$ for $15 \mathrm{sec}, 60^{\circ} \mathrm{C}$ for $1 \mathrm{~min}, 95^{\circ} \mathrm{C}$ for $15 \mathrm{sec}$ and a final extension step at $60^{\circ} \mathrm{C}$ for $15 \mathrm{sec}$. The expression levels of the target genes were determined following subtraction of the expression levels of GAPDH, with the value for the control group designated as 1 using the $2^{-\Delta \Delta \mathrm{Cq}}$ method (20).

Immunohistochemistry assay. Histological sections were heated for $60 \mathrm{~min}$ at $60^{\circ} \mathrm{C}$, deparaffinized through xylene and rinsed in a decreasing gradient of alcohol (100, 95 and 70\%). 
Table I. PCR primer sequences of BMP-7, Smad1, Smad5, and GAPDH.

\begin{tabular}{lll}
\hline Gene & \multicolumn{1}{c}{ Forward sequence } & \multicolumn{1}{c}{ Reverse sequence } \\
\hline BMP-7 & 5'-ATCCCCAATGTCTCACCACCTA-3' & 5'-AAGTATGCTGCTTATCAACCACG-3' \\
Smad1 & 5'-ATGAACTAAAGCCTCTGGAAT-3' & 5'-GGTTGTACTCGCTGTGCC-3' \\
Smad5 & 5'-AACCATGGGTTTGAGGCTGTG-3' & 5'-AGAGGCCCATGGAGGTGAATC-3' \\
GAPDH $^{\mathrm{a}}$ & 5'-GGCACAGTCAAGGCTGAGAATG-3' & 5'-ATGGTGGTGAAGACGCCAGTA-3'
\end{tabular}

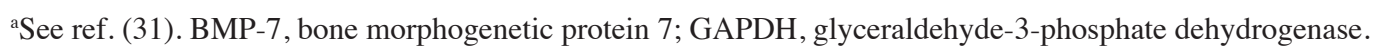

Subsequently, antigens were retrieved with citrate buffer (0.01 M; pH 6) in a microwave oven. Following treatment with hydrogen peroxide at $37^{\circ} \mathrm{C}$ for 30 min to block endogenous peroxidase activity, the sections were incubated with a primary antibody overnight at $4{ }^{\circ} \mathrm{C}$ and subsequently incubated with a peroxidase-conjugated affiniPure monoclonal goat anti-mouse or anti-rabbit immunoglobulin G secondary antibody (PV-9001 or PV-9002; Zhongshan Goldenbridge Biotechnology Co., Ltd., Beijing, China) for $30 \mathrm{~min}$ at room temperature. Finally, sections were stained with diaminobenzidine (Zhongshan Goldenbridge Biotechnology Co., Ltd.) for $1 \mathrm{~min}$, and subsequently counterstained with H\&E stain for a further $20 \mathrm{sec}$. The following primary antibodies were used: Monoclonal mouse anti-rat BMP-7 (1:200 dilution; cat. no. ab54904; Abcam, Cambridge, UK), polyclonal rabbit anti-rat collagen I (1:200 dilution; cat. no. ab34710; Abcam), polyclonal rabbit anti-rat $\alpha$-SMA (1:200 dilution; cat. no. ab5694; Abcam), polyclonal rabbit anti-rat TIMP-1 (1:200 dilution; cat. no. ab61224; Abcam) and monoclonal rabbit anti-rat p-Smad 1/5 (1:100 dilution; cat. no. 13820; Cell Signaling Technology, Inc., Danvers, MA, USA).

Westernblotting. Thetotal proteinwasextracted by homogenizing liver tissues in RIPA Lysis and Extraction buffer (Thermo Fisher Scientific) supplemented with a protease inhibitor cocktail from Roche (Summerville, NJ, USA). Subsequently, equal quantities of proteins were separated by $8-12 \%$ sodium dodecyl sulfate-polyacrylamide gel electrophoresis (SDS-PAGE) and transferred onto a nitrocellulose membrane. After blocking with $5 \%$ milk for $2 \mathrm{~h}$ at room temperature, the membranes were incubated with primary antibody (monoclonal mouse anti-rat BMP-7 (1:300 dilution; cat. no. ab54904; Abcam), monoclonal mouse anti-rat Smad 1/5 (1:300; cat. no. ab75273; Abcam), monoclonal rabbit anti-rat p-Smad 1/5 (1:300 dilution; cat. no. 13820; Cell Signaling Technology, Inc.), polyclonal rabbit anti-rat collagen I (1:300 dilution; cat. no. ab34710; Abcam), monoclonal mouse anti-rat collagen III (1:300 dilution; cat. no. ab6310; Abcam), polyclonal rabbit anti-rat $\alpha$-SMA (1:300 dilution; cat. no. ab5694; Abcam), polyclonal rabbit anti-rat TIMP-1 (1:300 dilution; cat. no. ab61224; Abcam) and polyclonal rabbit anti-rat $\beta$-actin (1:5,000 dilution; cat. no. ab25894; Abcam) overnight at $4^{\circ} \mathrm{C}$, and subsequently with secondary with secondary antibodies [peroxidase-conjugated affiniPure goat Anti-mouse (1:5,000 dilution; cat. no. ZB-2305) or anti-rabbit immunoglobulin G (1:5,000 dilution; cat. no. ZB-2301); both from Zhongshan Goldenbridge Biotechnology Co., Ltd.] at room temperature for $60 \mathrm{~min}$. The blots were visualized using an enhanced chemiluminescence kit
(Merck Millipore, Billerica, MA, USA). All primary antibodies were diluted 1:300, with the exception of $\beta$-actin $(1: 5,000)$.

Statistical analysis. The data are presented as the mean \pm standard deviation, unless otherwise specified. All statistical analyses were performed with SPSS 17.0 software (SPSS, Inc., Chicago, IL, USA). The differences between the means were analyzed using one-way analysis of variance and the least significant difference was used for multiple comparisons. $\mathrm{P}<0.05$ was considered to indicate a statistically significant difference.

\section{Results}

Cpd 861 reduces collagen deposition and tissue damage in the livers of rats induced by BDL. To evaluate the effects of Cpd 861 on BDL-induced hepatic fibrosis in rats, the histological changes of liver tissues were detected using H\&E and Masson staining. As shown in Fig. 1, the sham-operation group exhibited normal H\&E and Masson staining around the vessels, and a normal lobular architecture, with central veins and radiating hepatic cords. However, the BDL model group exhibited disturbed liver lobules characterized by a mess of deposition of fibrous tissue, which formed membrane-like intervals in the lobules of the liver, resulting in the formation of pseudo-lobules. By contrast, collagenous fibers were decreased, and pathological changes were markedly ameliorated, in the Cpd 861 treatment group. In addition, intrahepatic small bile duct proliferation was observed in the portal area in the BDL model group, although this was reduced in the Cpd 861 treatment group, indicating a role for Cpd 861 in the improvement of hepatic fibrosis.

Cpd 861 improves serum biochemical parameters in the BDL model rat. As shown in Fig. 2, when compared with the sham-operation group, BDL treatment significantly increased the serum levels of ALT, AST, TBIL and DBIL (all $\mathrm{P}<0.05)$. Although the addition of $\mathrm{Cps} 861$ exerted no significant effect on ALT and AST compared with the BDL treatment, it did elicit a significant reduction in the serum levels of TBIL and DBIL in the BDL model group $(\mathrm{P}<0.05)$, suggesting that $\mathrm{Cpd} 861$ attenuates cholestasis in the BDL model group.

Cpd 861 upregulates BMP-7/Smad signaling-associated genes in the BDL model rats. To assess the anti-fibrotic mechanism of Cpd 861, the effect of Cpd 861 on the expression of BMP-7/Smad signaling-associated genes was first assessed. When compared with the sham operation control group, the 

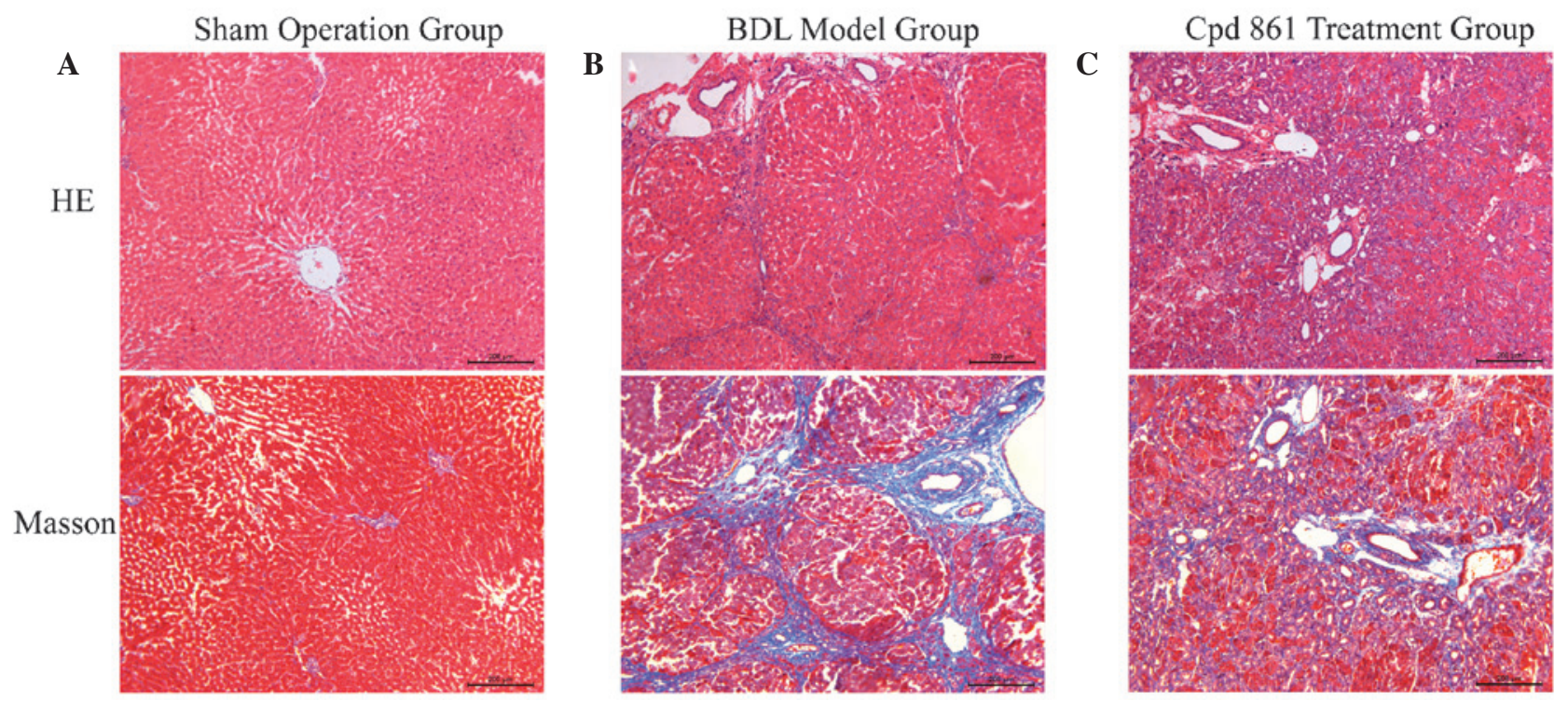

Figure 1. Histological examination of liver sections using HE and Masson staining (magnification, x50). Images are shown for the (A) sham operation group, (B) BDL model group and (C) Cpd 861 treatment group. BDL, bile duct ligation; Cpd 861, compound 861; HE, hematoxylin-eosin.
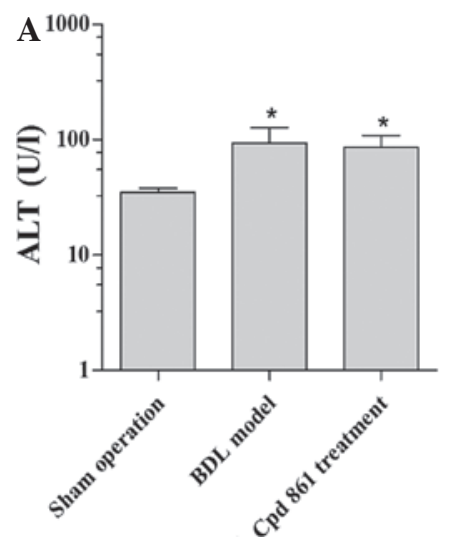
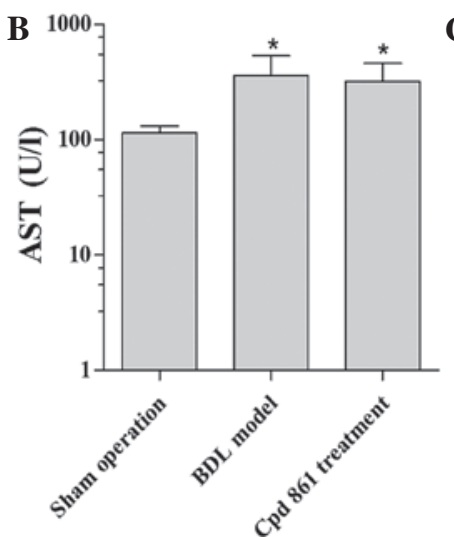
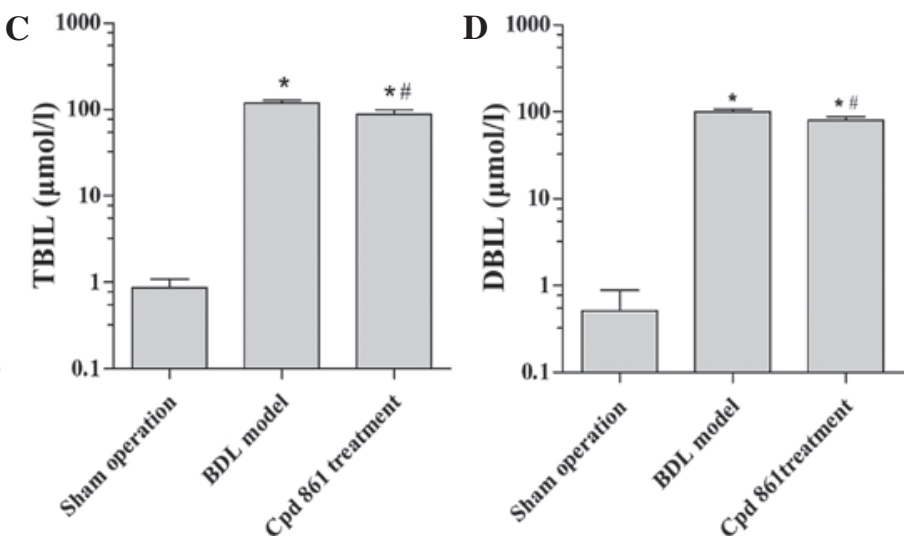

Figure 2. Effect of Cpd 861 on serum biochemical indicators. The levels of (A) ALT, (B) AST, (C) TBIL and (D) DBIL were assessed, and the data are expressed as the mean \pm standard deviation $(n=6) .{ }^{*} \mathrm{P}<0.05$ vs the sham operation group; ${ }^{*} \mathrm{P}<0.05$ vs. the BDL model group. ALT, alanine transaminase; AST, aspartate transaminase; TBIL, total bilirubin; DBIL: direct bilirubin; BDL, bile duct ligation; Cpd 861, compound 861.

BDL model group revealed an increased gene expression of Smad5, although no significant influence on the expression levels of BMP-7 and Smad1 were observed. When compared with the BDL model group, Cpd 861 treatment significantly increased the gene expression of BMP-7 and Smad5 $(\mathrm{P}<0.05)$, and modestly increased the expression of Smad1 $(\mathrm{P}>0.05$; Fig. 3).

It should be noted that the expression of $\operatorname{Smad} 8$ in the Cpd 861 treatment group was lower compared with that in the BDL model group (data not shown).

Cpd 861 reduces the expression of fibrosis-associated proteins, although it increases BMP-7/smad signaling, as detected by immunohistochemistry. To assess the mechanism by which Cpd 861 treatment attenuates hepatic fibrosis in BDL model rats, the expression and localization of several proteins involved in fibrosis formation and BMP-7/Smad signaling were further investigated. The expression levels of fibrosis-associated proteins, including collagen I, $\alpha$-SMA and TIMP1, were revealed to be negligible in the sham-operation group (Fig. 4A), although these proteins were observed in the BDL fibrosis model group (Fig. 4B). However, the expression levels of these fibrosis-associated proteins were downregulated on treatment with Cpd 861 (Fig. 4C), suggesting that Cpd 861 inhibits the progression of hepatic fibrosis. Furthermore, a negligible expression of BMP-7 and p-Smad1/5/8 was observed in the sham-operation and BDL model groups (Fig. 4A and B), which were, however, upregulated in the Cpd 861 treatment group (Fig. 4C), indicating upregulation of Cpd 861 in the BMP-7/Smad signaling pathway. Additionally, collagen I and TIMP1 were shown to exhibit positive immunostaining, predominantly in the hepatocytes and biliary epithelial cells, whereas $\alpha$-SMA was predominantly expressed in the mesenchymal region of the liver. Positive immunostaining of BMP-7 and $\mathrm{p}-\mathrm{Smad} 1 / 5 / 8$ were observed in the cytoplasm and nuclei of hepatocytes, but predominantly in the cytoplasm, indicating that Cpd 861 activates the BMP-7/Smad signaling pathway.

Cpd 861 reduces the expression of fibrosis-associated proteins, but enhances the BMP-7/Smad signaling pathway, 

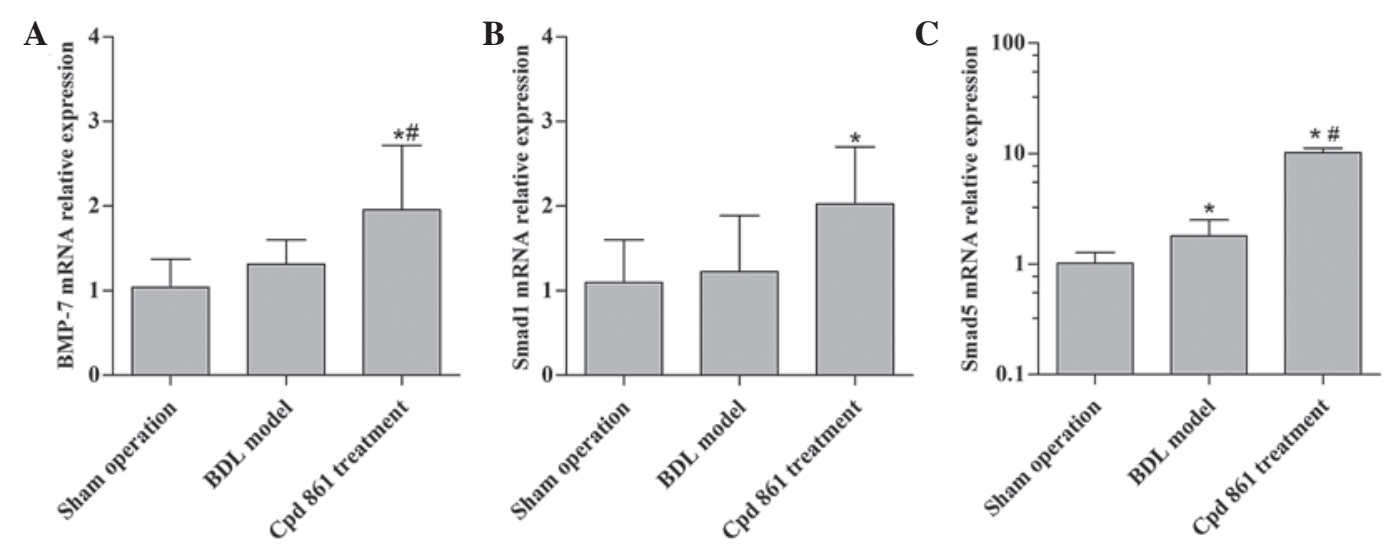

Figure 3. Effect of Cpd861 on the gene expression of (A) BMP-7, (B) Smad1 and (C) Smad5 in the BDL model rats detected by reverse transcription-quantitative polymerase chain reaction. The data are expressed as the mean \pm standard deviation $(n=6) .{ }^{*} \mathrm{P}<0.05$ vs. the sham operation group; ${ }^{*} \mathrm{P}<0.05$ vs. the $\mathrm{BDL}$ model group. BDL, bile duct ligation; Cpd 861, compound 861; BMP-7, bone morphogenetic protein 7.



Figure 4. Effect of Cpd 861 on the protein expression of collagen I, $\alpha$-SMA, TIMP1, BMP-7 and phosphorylated Smad1/5/8 analyzed by immunohistochemistry assay (magnification, x200). Representative images are shown for the (A) sham operation group, (B) BDL model group and (C) Cpd 861 treatment group. $\alpha$-SMA, $\alpha$-smooth muscle actin; TIMP1, tissue inhibitor of metalloproteinase 1; BMP-7, bone morphogenetic protein 7; BDL, bile duct ligation; Cpd 861, compound 861 . 


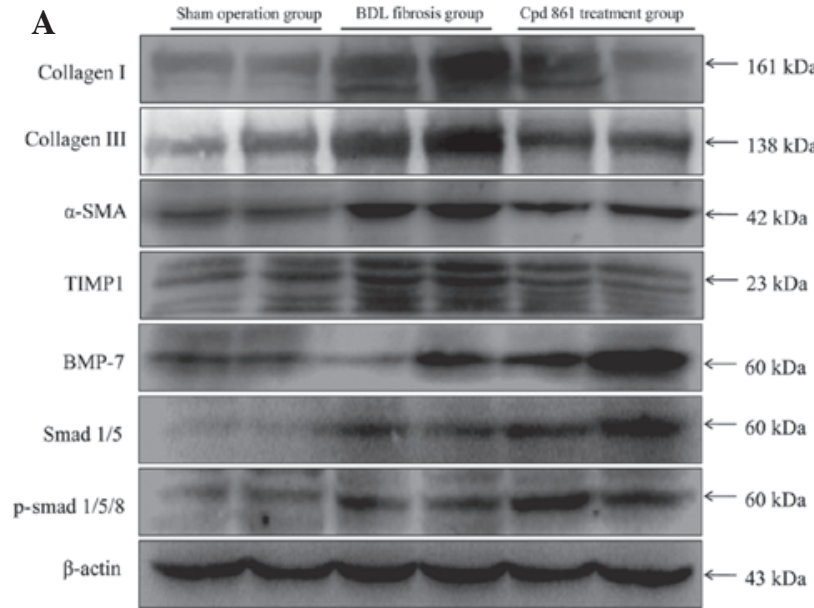

D

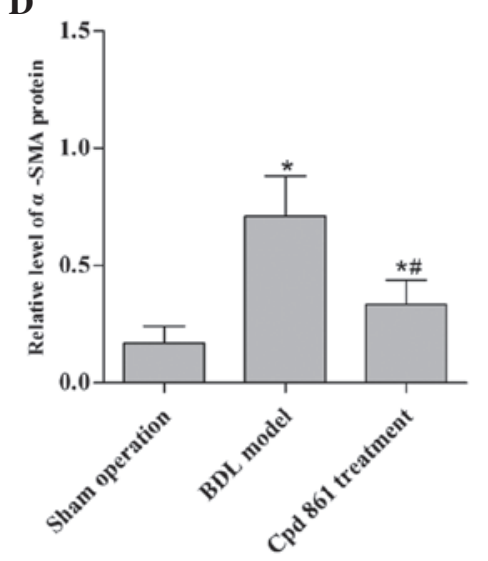

G

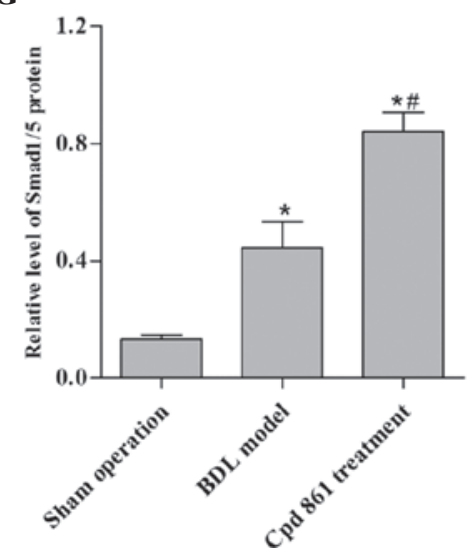

B

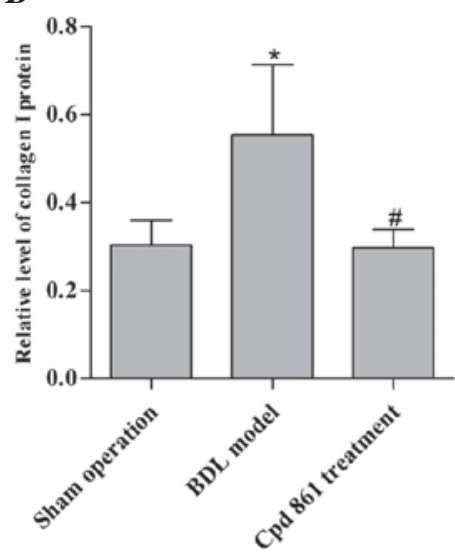

$\mathbf{E}$

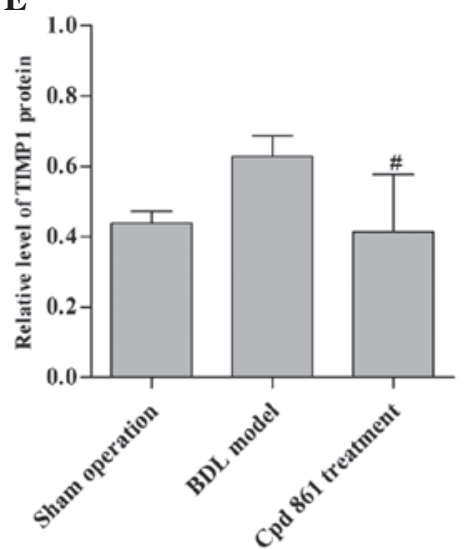

H

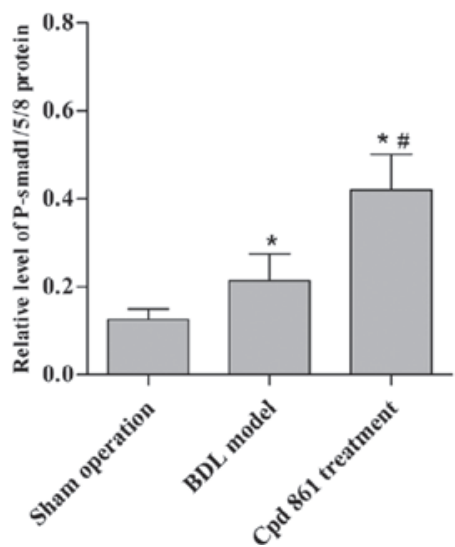

C

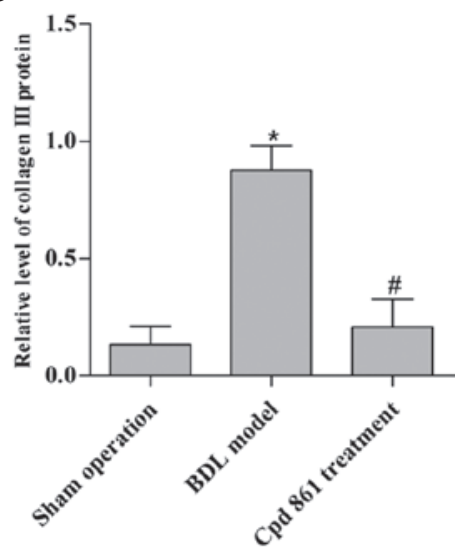

F

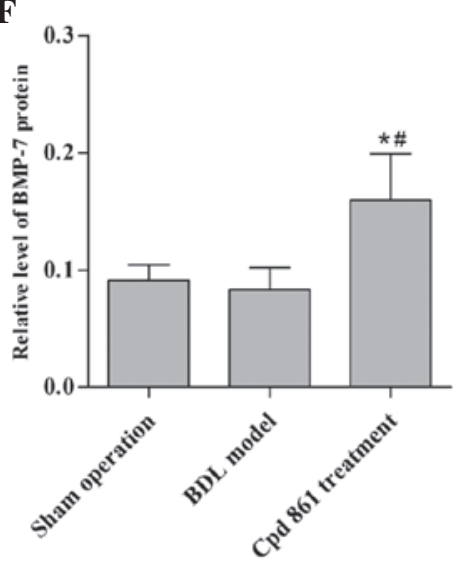

Figure 5. Protein expression levels of collagen I, collagen III, $\alpha$-SMA, TIMP1, BMP-7 and p-Smad1/5/8 were (A) evaluated by western blotting and were normalized to the levels of $\beta$-actin. Quantification of the identical data is shown for (B) collagen I, (C) collagen III, (D) $\alpha$-SMA, (E) TIMP1, (F) BMP-7, (G) Smad 1/5 and (H) $\mathrm{p}-\mathrm{Smad} 1 / 5 / 8$. The data are expressed as the mean \pm standard deviation $(\mathrm{n}=6) .{ }^{*} \mathrm{P}<0.05$ vs. the sham operation group; ${ }^{*} \mathrm{P}<0.05$ vs. the BDL model group. $\alpha-\mathrm{SMA}$, $\alpha$-smooth muscle actin; TIMP1, tissue inhibitor of metalloproteinase 1; BMP-7, bone morphogenetic protein 7; BDL, bile duct ligation; Cpd 861, compound 861.

as detected by western blotting. As shown in Fig. 5A-E, BDL induced the expression of collagen I, collagen III, $\alpha$-SMA, and TIMP1, although Cpd 861 reversed the BDL effect, indicating that $\mathrm{Cpd} 861$ was effective at completely inhibiting the development of cholestatic hepatic fibrosis. In addition, BDL induced the expression of Smad1/5 and p-Smad1/5/8, although no significant differences in terms of the protein expression levels of BMP-7 were observed for the BDL model group (Fig. 5F-H). Cpd 861 treatment further upregulated the expression of Smad1/5 and p-Smad1/5/8, as well as increasing the protein expression levels of BMP-7 compared with the
BDL model group. These data further demonstrated that the BMP-7/Smad signaling pathway is activated during the process of administering Cpd 861 for hepatic fibrosis treatment.

\section{Discussion}

Hepatic fibrosis is a complex pathophysiological process, involving various growth factors and their underlying signaling networks. It should be noted that the TGF- $\beta$ signaling pathway is considered to be an activator of HSCs, and exerts a crucial role in fibrogenesis (21). Once activated, HSCs acquire the 
capacity to proliferate, and to secrete $\alpha$-SMA and various other chemoattractant factors. Additionally, activated HSCs exhibit an altered expression profile of collagens, MMPs and TIMPs (22). In accordance with our data, increased protein expression levels of collagen I, collagen III, $\alpha$-SMA and TIMP1 were observed during BDL-induced fibrogenesis.

Cpd 861 has been confirmed by liver biopsies to be an effective compound for the treatment of hepatic fibrosis. Several studies have revealed that $\mathrm{Cpd} 861$ inhibits the expression of collagen synthesis-associated genes and the MMP-1 gene $(15,23,24)$, and that the activation and proliferation of HSCs is inhibited (25). In the present study, Cpd 861 treatment was observed to reduce the levels of ALT, AST, TBIL and DBIL, suggesting a protective effect of Cpd 861 in liver function and a beneficial role in alleviating cholestasis. The $\mathrm{H} \& \mathrm{E}$ and Masson staining experiments confirmed that $\mathrm{Cpd} 861$ ameliorated the pathological changes of the liver, decreased the synthesis of collagenous fiber, reduced intrahepatic small bile duct proliferation and reduced the levels of apoptosis in the hepatocytes. Furthermore, the protein expression levels of collagen I, collagen III, $\alpha$-SMA and TIMP1 were decreased following Cpd 861 treatment. Taken together, these results have confirmed that Cpd 861 is a potentially useful anti-fibrotic agent.

On the other hand, BMP-7 is one member of the BMP family that is expressed in a number of organized regions of the early embryo, and was originally described in terms of its ability to accelerate bone formation (26). Subsequently, a number of studies have demonstrated that BMP-7 possesses anti-inflammatory and anti-fibrotic properties in the fibrotic model (10), and is able to attenuate hepatic fibrosis by negatively regulating the functions of TGF- $\beta 1(11,12,27)$. Studies have also indicated that the actions of BMP-7 are transduced by Smad1, Smad5 and Smad8, which subsequently form a complex with Smad4 to enter the nucleus to regulate gene expression, whereas Smad2 and Smad3 regulate the activity of TGF- $\beta 1$ (28-30). On the basis of the above-mentioned evidence, in the present study a rat BDL model was used to determine the mechanism(s) via which Cpd 861 regulates the fibrotic process, and the results have revealed that the gene and protein expression levels of components of the BMP-7/Smad signaling pathway were elevated following treatment with Cpd 861. In addition, BMP-7 and p-Smad1/5/8 were observed in the cytoplasm and nuclei of the hepatocytes. These results indicated that the BMP-7/Smad1/5/8 signaling pathway was activated on treatment with Cpd 861 .

Nevertheless, the present study had certain limitations. Further studies are required to evaluate the effects of different dosages and durations of Cpd 861 treatment on the BMP-7/Smad signaling pathway induced by BDL, and to clarify the possible underlying molecular mechanisms.

In conclusion, we have demonstrated that an attenuation of hepatic fibrosis and an amelioration of liver function were detected in the Cpd 861-treated group, as indicated by decreased protein levels of collagen I, collagen III, $\alpha$-SMA and TIMP1. Furthermore, elevated protein expression levels of BMP-7, Smad1/5 and p-Smad1/5/8 were observed following treatment with Cpd 861 during hepatic fibrosis. Therefore, our collected data support the concept that $\mathrm{Cpd} 861$ attenuates hepatic fibrosis via the upregulation of BMP-7/Smad signaling.

\section{Acknowledgements}

This study was supported by the National Natural Science Foundation of China [no. 81341090] and the WBE Liver Fibrosis Foundation [no. CFHPC20120145]. We would also like to thank Medjaden Bioscience Limited for assisting in the preparation of this paper.

\section{References}

1. Lim YS and Kim WR: The global impact of hepatic fibrosis and end-stage liver disease. Clin Liver Dis 12: 733-746, vii, 2008.

2. Moreira RK: Hepatic stellate cells and liver fibrosis. Arch Pathol Lab Med 131: 1728-1734, 2007.

3. Brenner DA, Waterboer T, Choi SK, Lindquist JN, Stefanovic B, Burchardt E, Yamauchi M, Gillan A and Rippe RA: New aspects of hepatic fibrosis. J Hepatol 32 (Suppl 1): S32-S38, 2000.

4. Okuno M, Akita K, Moriwaki H, Kawada N, Ikeda K, Kaneda K, Suzuki Y and Kojima S: Prevention of rat hepatic fibrosis by the protease inhibitor, camostat mesilate, via reduced generation of active TGF- $\beta$. Gastroenterology 120: 1784-1800, 2001.

5. Ling H, Roux E, Hempel D, Tao J, Smith M, Lonning S, Zuk A Arbeeny $\mathrm{C}$ and Ledbetter $\mathrm{S}$ : Transforming growth factor $\beta$ neutralization ameliorates pre-existing hepatic fibrosis and reduces cholangiocarcinoma in thioacetamide-treated rats. PLoS One 8: e54499, 2013

6. Yata Y, Gotwals P, Koteliansky V and Rockey DC: Dose-dependent inhibition of hepatic fibrosis in mice by a TGF- $\beta$ soluble receptor: Implications for antifibrotic therapy. Hepatology 35: 1022-1030, 2002.

7. Reddi AH: Role of morphogenetic proteins in skeletal tissue engineering and regeneration. Nat Biotechnol 16: 247-252, 1998.

8. Huo L, Liu K, Pei J, Yang Y, Ye Y, Liu Y, Sun J, Han H, Xu W and Gao Y: Fluoride promotes viability and differentiation of osteoblast-like Saos-2 cells via BMP/Smads signaling pathway. Biol Trace Elem Res 155: 142-149, 2013.

9. Kretzschmar M and Massagué J: SMADs: Mediators and regulators of TGF- $\beta$ signaling. Curr Opin Genet Dev 8: 103-111, 1998.

10. Wang SL, Yang CQ, Qi XL, Yuan M, Chang YZ, Yang L and Gao HJ: Inhibitory effect of bone morphogenetic protein-7 on hepatic fibrosis in rats. Int J Clin Exp Pathol 6: 897-903, 2013.

11. Chen BL, Peng J, Li QF, Yang M, Wang Y and Chen W: Exogenous bone morphogenetic protein-7 reduces hepatic fibrosis in Schistosoma japonicum-infected mice via transforming growth factor- $\beta /$ Smad signaling. World J Gastroenterol 19: 1405-1415, 2013.

12. Zeisberg M, Hanai J, Sugimoto H, Mammoto T, Charytan D, Strutz F and Kalluri R: BMP-7 counteracts TGF-beta1-induced epithelial-to-mesenchymal transition and reverses chronic renal injury. Nat Med 9: 964-968, 2003.

13. Kinoshita K, Iimuro Y, Otogawa K, Saika S, Inagaki Y, Nakajima Y, Kawada N, Fujimoto J, Friedman SL and Ikeda K: Adenovirus-mediated expression of BMP-7 suppresses the development of liver fibrosis in rats. Gut 56: 706-714, 2007.

14. Yin SS, Wang BE, Wang TL, Jia JD and Qian LX: The effect of Cpd 861 on chronic hepatitis B related fibrosis and early cirrhosis: A randomized, double blind, placebo controlled clinical trial. Zhonghua Gan Zang Bing Za Zhi 12: 467-470, 2004 (In Chinese).

15. Wang L, Wang BE, Wang J, Xiao PG and Tan XH: Herbal compound 861 regulates mRNA expression of collagen synthesis- and degradation-related genes in human hepatic stellate cells. World J Gastroenterol 14: 1790-1794, 2008.

16. Wang L, Wang J, Wang BE, Xiao PG, Qiao YJ and Tan XH: Effects of herbal compound 861 on human hepatic stellate cell proliferation and activation. World J Gastroenterol 10: 2831-2835, 2004.

17. Aghaei I, Shabani M, Doustar N, Nazeri M and Dehpour A: Peroxisome proliferator-activated receptor- $\gamma$ activation attenuates motor and cognition impairments induced by bile duct ligation in a rat model of hepatic cirrhosis. Pharmacol Biochem Behav 120: 133-139, 2014.

18. Javadi-Paydar M, Ghiassy B, Ebadian S, Rahimi N, Norouzi A and Dehpour AR: Nitric oxide mediates the beneficial effect of chronic naltrexone on cholestasis-induced memory impairment in male rats. Behav Pharmacol 24: 195-206, 2013. 
19. Uchinami H, Seki E, Brenner DA and D'Armiento J: Loss of MMP 13 attenuates murine hepatic injury and fibrosis during cholestasis. Hepatology 44: 420-429, 2006.

20. Livak KJ and Schmittgen TD: Analysis of relative gene expression data using real-time quantitative PCR and the 2(-Delta Delta C(T)) Method. Methods 25: 402-408, 2001

21. Shen H, Huang G, Hadi M, Choy P, Zhang M, Minuk GY, Chen Y and Gong Y: Transforming growth factor-betal downregulation of Smad1 gene expression in rat hepatic stellate cells. Am J Physiol Gastrointest Liver Physiol 285: G539-G546, 2003.

22. Yang T, Chen SL, Lu XJ, Shen CY, Liu Y and Chen YP: Bone morphogenetic protein 7 suppresses the progression of hepatic fibrosis and regulates the expression of gremlin and transforming growth factor $\beta 1$. Mol Med Rep 6: 246-252, 2012.

23. Ding HG, Tang SZ, Wang BE, Jia JD and Zhao CH: Effects of herbal compound 861 on hepatic stellate cell expressing endothelin-1 protein and mRNA. Chin J Hepatol 11: 308, 2003 (In Chinese).

24. Yin C, Ma H, Wang A, Ma X, Jia J and Wang B: Effect of compound 861 on tissue inhibitor of metalloproteinase 1 gene expression of HSC-T6 cells. Chin J Hepatol 10: 197-199, 2002 (In Chinese).
25. You H, Wang B and Wang T: Proliferation and apoptosis of hepatic stellate cells and effects of compound 861 on liver fibrosis. Chin J Hepatol 8: 78-80, 2000 (In Chinese).

26. Cheifetz S, Li IW, McCulloch CA, Sampath K and Sodek J: Influence of osteogenic protein-1 (OP-1;BMP-7) and transforming growth factor-beta 1 on bone formation in vitro. Connect Tissue Res 35: 71-78, 1996.

27. Wang LP, Dong JZ, Xiong LJ, Shi KQ, Zou ZL, Zhang SN, Cao ST, Lin Z and Chen YP: BMP-7 attenuates liver fibrosis via regulation of epidermal growth factor receptor. Int J Clin Exp Pathol 7: 3537-3547, 2014

28. Heldin $\mathrm{CH}$, Miyazono K and ten Dijke P: TGF-beta signalling from cell membrane to nucleus through SMAD proteins. Nature 390: 465-471, 1997.

29. Wrana JL: Regulation of Smad activity. Cell 100: 189-192, 2000

30. Wang S and Hirschberg R: Bone morphogenetic protein-7 signals opposing transforming growth factor beta in mesangial cells. J Biol Chem 279: 23200-23206, 2004 